\title{
Niemann-Pick Disease, Type B
}

National Cancer Institute

\section{Source}

National Cancer Institute. Niemann-Pick Disease, Type B. NCI Thesaurus. Code C126866.

An autosomal recessive lysosomal storage disease caused by mutations in the SMPD1 gene, encoding sphing omyelin phosphodiesterase. The condition is characterized by hepatosplenomegaly and interstitial lung disease, but with little neurological involvement. It is part of a continuum of disease resulting from decrease activity of sphing omyelin phosphodiesterase, with Type B being the milder form. 